\title{
Accesibilidad en los sitios web empresariales de la provincia de Loja. Análisis preliminar
}
Accessibility in the business websites of the province of Loja. Preliminary analysis

\section{Acessibilidade nos sites de negócios da província de Loja. Análise Preliminar}

Diego Germán Salas-Paucar ${ }^{\text {I }}$

diego.sp28@gmail.com

Milton Alfredo Campoverde-Molina II

cencalada@ucacue.edu.ec

Recibido: 28 de abril de 2019 *Aceptado: 10 de junio de 2019 * Publicado: 05 de julio de 2019

ı. Ingeniero en Electrónica y Telecomunicaciones, Estudiante, Jefatura de Posgrados. Universidad Católica de Cuenca, Cuenca, Ecuador.

I. Ingeniero en Sistemas, Docente de la Unidad Académica de Tecnologías de la Información, Jefatura de Posgrados, Universidad Católica de Cuenca, Cuenca, Ecuador. 


\section{Resumen}

En este artículo se presentan los resultados del análisis preliminar de accesibilidad de los sitios web empresariales de la provincia de Loja, Ecuador. El análisis se lo hizo en base a las Pautas de Accesibilidad para el Contenido Web (WCAG) 2.0 con un nivel de conformidad AA. Para este fin se identificaron 46 sitios web empresariales los cuales usan el dominio.com y dominio.com.ec. De cada portal web se seleccionó las 4 páginas más representativas para un total de 184 páginas web analizadas. Para el análisis del porcentaje de cumplimiento en las páginas de inicio se utilizó las herramientas en línea OAW y AccessMonitor, y para determinar los errores en las páginas seleccionadas de los portales web se utilizó TAW, WAVE, CSS Validation Services y Markup Validation Service. Con base a los resultados obtenidos se concluye que; el nivel de accesibilidad de las páginas web es bajo y requiere corregir errores y así cumplir con el reglamento técnico ecuatoriano RTE INEN 288, que establece que los sitios web ecuatorianos que proporcionan un servicio público deben ser accesibles WCAG 2.0 nivel AA hasta el 8 de agosto de 2020 .

Palabras clave: Accesibilidad; Web; discapacidad; WCAG 2.0; compañías.

\section{Abstract}

This article presents the results of the preliminary analysis of accessibility of business websites in the province of Loja, Ecuador. The analysis was done based on the Accessibility Guidelines for Web Content (WCAG) 2.0 with an AA compliance level. To this end, 46 business websites were identified which use domain.com and domain.com.ec. From each web portal, the 4 most representative pages were selected for a total of 184 web pages analyzed. For the analysis of the percentage of compliance in the home pages, the online tools OAW and AccessMonitor were used, and to determine the errors in the selected pages of the web portals, TAW, WAVE, CSS Validation Services and Markup Validation Service were used. Based on the results obtained, it is concluded that; the level of accessibility of web pages is low and requires correcting errors and thus comply with the Ecuadorian technical regulation RTE INEN 288, which establishes that Ecuadorian websites that provide a public service must be accessible WCAG 2.0 AA level until August 8 from 2020.

Key words: Web; Accessibility; Disability; WCAG 2.0; Companies.

\footnotetext{
\begin{tabular}{|l|l|}
213 & Vol. 5, núm. 3, julio, 2019, pp.212-237 \\
Diego German Salas Paucar, Milton Alfredo Campoverde Molina
\end{tabular}
} 


\section{Resumo}

Este artigo apresenta os resultados da análise preliminar da acessibilidade de sites de negócios na província de Loja, no Equador. A análise foi feita com base nas Diretrizes de Acessibilidade para Conteúdo da Web (WCAG) 2.0 com um nível de conformidade AA. Para este fim, foram identificados 46 sites de negócios que usam domain.com e domain.com.ec. De cada portal da web, as 4 páginas mais representativas foram selecionadas para um total de 184 páginas analisadas. Para a análise da percentagem de conformidade homepages ferramentas utilizadas OAW online e AccessMonitor, e para determinar os erros em páginas selecionadas do Portais TAW, WAVE, foi usado CSS validação Serviços e Markup Validation Service. Com base nos resultados obtidos, conclui-se que; o nível de acessibilidade das páginas da web é baixo e requer a correção de erros e, portanto, está em conformidade com o regulamento técnico equatoriano RTE INEN 288, que estabelece que os sites equatorianos que fornecem serviço público devem estar acessíveis no nível WCAG 2.0 AA até 8 de agosto. a partir de 2020.

Palavras-chave: Acessibilidade; Web; Deficiência WCAG 2.0; Empresas.

\section{Introducción}

La accesibilidad tiene como fin, que las personas con algún tipo de discapacidad puedan hacer uso de las páginas web con el menor esfuerzo. Es muy importante que la Web sea accesible para así proporcionar un acceso equitativo e igualdad de oportunidades a las personas con discapacidad (Campoverde \& Valverde, 2019). El acceso a la información a través de tecnologías como la web es conveniente para algunos, pero para las personas con discapacidades es cada vez más lo que hace posible las oportunidades educativas y de empleo (World Wide Web Consortium, 1997).

Las pautas de accesibilidad para el contenido web WCAG 2.0, fueron aprobadas como estándar ISO/IEC40500:2012 el 15 de octubre de 2012. Seguir estas pautas puede a menudo ayudar a que el contenido Web sea más usable para cualquier tipo de usuario (World Wide Web Consortium, 2008b). Los sitios, las tecnologías o las herramientas que no cumplan con estándares pueden crear barreras que excluyen a las personas del uso de la Web (Acosta \& Luja-Mora, 2017).

\footnotetext{
\begin{tabular}{|l|l|}
214 & Vol. 5, núm. 3, julio, 2019, pp.212-237 \\
Diego German Salas Paucar, Milton Alfredo Campoverde Molina
\end{tabular}
} 
En Ecuador, el 28 de enero de 2014 se publicó en el Registro Oficial № 171 la aprobación de la norma NTE INEN-ISO/IEC 40500 "Tecnología de la información - Directrices de accesibilidad para el contenido web del W3C (WCAG) 2.0 (ISO/IEC 40500:2012, IDT)" (Servicio Ecuatoriano de Normalización, 2014) y entro en vigor el 8 de agosto del 2016. Para realizar el seguimiento del cumplimiento de la Norma NTE INEN-ISO/IEC 40500 se creó en Ecuador el reglamento técnico ecuatoriano RTE INEN 288 "Accesibilidad para el contenido web" (Servicio Ecuatoriano de Normalización, 2016). En la primera transitoria de la norma NTE INEN el plazo para adecuar los sitios web a nivel de conformidad WCAG 2.0 "A" es de 2 años a partir del 8 de agosto de 2016. En la segunda Transitoria de la norma NTE INEN el plazo para adecuar los sitios web a nivel de conformidad WCAG 2.0 "AA" es 4 años a partir del 8 de agosto del 2016.

La industria ha venido evolucionando en las últimas décadas, y esta evolución va de la mano de las innovaciones técnicas que han cambiado la forma de la empresa. Desde el año 1800 cuando se dio la primera revolución industrial, hasta la actualidad en la que se vive la cuarta revolución industrial, se ha pasado de la introducción de equipos de producción mecánicos impulsados por agua y la energía del vapor al uso de sistemas cibernéticos. Es importante que en esta nueva era industrial las compañías dispongan de un portal web para que no desaprovechen la oportunidad de utilizar este gran recurso que es el internet. Además, los sitios web tienen que cumplir las directrices de accesibilidad establecidas en la Norma NTE INEN-ISO/IEC 40500.

Actualmente la población en Ecuador es de 17.266.684 (Instituto Nacional de Estadística y Censos, 2019), y existente un total de 461.687 personas registradas que poseen algún tipo de discapacidad (Consejo Nacional para la Igualdad de Discapacidades, 2019). Esto representa un total del 2.7\% de la población ecuatoriana que presenta algún tipo de discapacidad. La IUT (Unión Internacional de Telecomunicaciones) en su artículo de accesibilidad para todos menciona; que las personas con discapacidad de todo el mundo deben poder disfrutar de los mismos derechos y oportunidades que todos los demás, llevar a cabo su vida como ciudadanos de pleno derecho y aportar una valiosa contribución a la sociedad (Unión Internacional de Telecomunicaciones, 2009). En Ecuador se debe cumplir con los establecido en la normativa NTE INEN-ISO/IEC 40500 para de garantizar la accesibilidad a todos los ciudadanos por igual.

\footnotetext{
\begin{tabular}{|l|l}
215 & Vol. 5, núm. 3, julio, 2019, pp.212-237 \\
Diego German Salas Paucar, Milton Alfredo Campoverde Molina
\end{tabular}
} 
En la provincia de Loja existen 14,933 personas con discapacidades registradas (Consejo Nacional para la Igualdad de Discapacidades, 2019), de un total de 448,966 habitantes (Instituto Nacional de Estadística y Censos, 2019). Loja es uno de los más importantes centros administrativos, económicos, financieros y comerciales del sur de Ecuador. Desde el 2007, Loja le está apostando a desarrollar la industria de tecnologías, puntualmente en el ámbito del software accesible vía Internet. Es importante esta investigación porque, a partir de los datos aquí presentados las empresas pueden verificar como está el tema de cumplimiento de la normativa NTE INENISO/IEC 40500 en sus portales web, y corregir los errores que están presentes en ellos.

Esta investigación tiene como objetivo realizar un análisis de accesibilidad con las WCAG 2.0 con un nivel de conformidad nivel AA de las páginas web empresariales de la provincia de Loja. Con este análisis se busca dar respuestas a las siguientes interrogantes.

- ¿Cuantas empresas domiciliadas en la provincia de Loja cuentan con un sitio web empresarial?

- ¿Cuántos de los sitios web empresariales son gestionados por un gestor de contenidos CMS?

- ¿Cuál es el promedio de errores de en los sitios web de las empresas de la provincia de Loja?

- ¿Los sitios web de las empresas de la provincia de Loja cumplen la normativa NTE INENISO/IEC 40500 con un nivel de conformidad "AA"?

La estructura del artículo presenta en la sección 2 conceptos relacionados con la investigación. En la sección 3 se trata de trabajos relacionados con la investigación. En la sección 4 se expone la metodología utilizada en la investigación. En la sección 5 se presentan los resultados de la investigación. En la sección 6 se presentan las conclusiones de la investigación con base a los resultados obtenidos.

\footnotetext{
\begin{tabular}{|l|l|}
216 & Vol. 5, núm. 3, julio, 2019, pp.212-237 \\
Diego German Salas Paucar, Milton Alfredo Campoverde Molina
\end{tabular}
} 


\section{Desarrollo}

\section{Pautas de Accesibilidad para el Contenido Web (WCAG) 2.0.}

Las WCAG 2.0 cubren un amplio rango de recomendaciones para crear contenido Web más accesible (World Wide Web Consortium, 2008a). El cumplimiento de estas pautas garantiza que un mayor número de personas con o sin discapacidades puedan hacer uso de las páginas web. La W3C da las pautas WCAG 2.0 (World Wide Web Consortium, 2008a), estas se componen de 4 principios, 12 pautas y 61 criterios de cumplimiento (éxito), más un número no determinado de técnicas suficientes y técnicas de asesoramiento (Lujan-Mora, 2019). En la tabla 1 se muestra los principios con sus respectivas pautas de accesibilidad web.

Tabla 1. Principios y descripción de pautas de accesibilidad.

\begin{tabular}{|c|c|c|c|}
\hline \multirow{6}{*}{ Perceptible } & Pauta 1.1 & $\begin{array}{l}\text { Alternativas } \\
\text { Textuales }\end{array}$ & $\begin{array}{l}\text { Proporcionar alternativas textuales } \\
\text { para todo contenido no textual de } \\
\text { modo que se pueda convertir a otros } \\
\text { formatos que las personas necesiten, } \\
\text { tales como textos ampliados, braille, } \\
\text { voz, símbolos o en un lenguaje más } \\
\text { simple. }\end{array}$ \\
\hline & Pauta 1.2 & $\begin{array}{l}\text { Contenido } \\
\text { multimedia } \\
\text { independiente del } \\
\text { tiempo }\end{array}$ & $\begin{array}{l}\text { Medios tempodependientes: } \\
\text { proporcionar alternativas para los } \\
\text { medios tempodependientes. }\end{array}$ \\
\hline & Pauta 1.3 & Adaptable & $\begin{array}{l}\text { Crear contenido que pueda } \\
\text { presentarse de diferentes formas (por } \\
\text { ejemplo, con una disposición más } \\
\text { simple) sin perder información o } \\
\text { estructura. }\end{array}$ \\
\hline & Pauta 1.4 & Distinguible & $\begin{array}{l}\text { Facilitar a los usuarios ver y oír el } \\
\text { contenido, incluyendo la separación } \\
\text { entre el primer plano y el fondo. }\end{array}$ \\
\hline & Pauta 2.1 & Teclado accessible & $\begin{array}{l}\text { Proporcionar acceso a toda la } \\
\text { funcionalidad mediante el teclado. }\end{array}$ \\
\hline & Pauta 2.2 & Tiempo suficiente & $\begin{array}{l}\text { Proporcionar a los usuarios el tiempo } \\
\text { suficiente para leer y usar el } \\
\text { contenido. }\end{array}$ \\
\hline
\end{tabular}

\footnotetext{
\begin{tabular}{l|l}
217 & Vol. 5, núm. 3, julio, 2019, pp.212-237 \\
Diego German Salas Paucar, Milton Alfredo Campoverde Molina
\end{tabular}
} 


\begin{tabular}{|c|c|c|c|}
\hline \multirow[t]{2}{*}{ Operable } & Pauta 2.3 & Ataques epilépticos & $\begin{array}{l}\text { No diseñar contenido de un modo } \\
\text { que se sepa podría provocar ataques, } \\
\text { espasmos o convulsiones. }\end{array}$ \\
\hline & Pauta 2.4 & Navegación & $\begin{array}{l}\text { Proporcionar medios para ayudar a } \\
\text { los usuarios a navegar, encontrar } \\
\text { contenido y determinar dónde se } \\
\text { encuentran. }\end{array}$ \\
\hline \multirow{3}{*}{ Comprensible } & Pauta 3.1 & Legible & $\begin{array}{l}\text { Hacer que los contenidos textuales } \\
\text { resulten legibles y comprensibles. }\end{array}$ \\
\hline & Pauta 3.2 & Previsible & $\begin{array}{l}\text { Hacer que las páginas web aparezcan } \\
\text { y operen de manera predecible. }\end{array}$ \\
\hline & Pauta 3.3 & $\begin{array}{l}\text { Asistencia a la } \\
\text { entrada de datos }\end{array}$ & $\begin{array}{l}\text { Ayudar a los usuarios a evitar y } \\
\text { corregir los errores. }\end{array}$ \\
\hline Robusto & Pauta 4.1 & Comprensible & $\begin{array}{l}\text { Maximizar la compatibilidad con las } \\
\text { aplicaciones de usuario actuales y } \\
\text { futuras, incluyendo las ayudas } \\
\text { técnicas. }\end{array}$ \\
\hline
\end{tabular}

Fuente: (Luján-Mora, 2019a)

\section{Conformidad}

Para que una página web se ajuste a WCAG 2.0, se deben cumplir los siguientes requisitos de conformidad (World Wide Web Consortium, 2008a):

Nivel de conformidad: Uno de los siguientes niveles de conformidad se satisface por completo.

Nivel A: Para el nivel A de conformidad (el mínimo nivel de conformidad), la página web satisface todos los criterios de éxito de nivel A, o se proporciona una versión alternativa conforme.

Nivel AA: Para el nivel AA de conformidad, la página web satisface todos los criterios de éxito de nivel A y AA, o se proporciona una versión alternativa conforme al nivel AA.

Nivel AAA: Para el nivel AAA de conformidad, la página web satisface todos los criterios de éxito de nivel A, AA y AAA, o se proporciona una versión alternativa conforme al nivel AAA.

Si un autor desea hacer una declaración de conformidad, entonces tal declaración debe incluir la siguiente información (World Wide Web Consortium, 2008a):

- $\quad$ Fecha de la declaración

\begin{tabular}{|l|l}
218 & Vol. 5, núm. 3, julio, 2019, pp.212-237 \\
Diego German Salas Paucar, Milton Alfredo Campoverde Molina
\end{tabular} 
- $\quad$ Título de las Pautas, versión y URI "WCAG 2.0 "

- $\quad$ Nivel de conformidad satisfecho: (Nivel A, AA o AAA)

- Una descripción concisa de las páginas web, tal como una lista de sus URIs para las que se hace la declaración, incluyendo cualesquiera subdominios a los que abarque la declaración.

\section{Norma Ecuatoriana de Accesibilidad Web.}

En Ecuador el 28 de enero de 2014 el Servicio Ecuatoriano de Normalización publicó en el registro Oficial $N^{\circ} 171$ la aprobación de la norma NTE INEN-ISO/IEC 40500 "Tecnología de la información - Directrices de accesibilidad web del W3C (WCAG) 2.0 (ISO/IEC 40500:2012, IDT)" (Servicio Ecuatoriano de Normalización, 2014). El 10 de febrero de 2016 el en el Registro Oficial No. 686 se encuentra aprobado el Reglamento Técnico Ecuatoriano RTE INEN 288 “accesibilidad para el contenido web" (Servicio Ecuatoriano de Normalización, 2016), éste reglamento permite realizar el seguimiento del cumplimiento obligatorio en el Ecuador de la Norma NTE INEN-ISO/IEC 40500. Entre sus requisitos establece:

- $\quad$ Aplica a todos los contenidos web en el sector público y privado que presten servicios públicos.

- $\quad$ Se especifica que se debe satisfacer por completo el nivel de conformidad AA establecido en la norma NTE INEN-ISO/IEC 40500.

- $\quad$ El cumplimiento de este reglamento se debe demostrar colocando en el propio sitio web el certificado de conformidad de primera parte.

Además, se establece dos disposiciones transitorias en donde se fija el plazo para el cumplimiento del reglamento y la norma:

- Transitoria Primera. Los propietarios de los sitios web a los que se aplica este reglamento técnico tendrán un plazo de 2 años para adecuar sus sitios web existentes al momento de entrar en vigencia el reglamento de acuerdo al nivel de conformidad A de la norma NTE INEN vigente. El

\footnotetext{
\begin{tabular}{|l|l|l|}
219 & Vol. 5, núm. 3, julio, 2019, pp.212-237 \\
Diego German Salas Paucar, Milton Alfredo Campoverde Molina
\end{tabular}
} 
8 de agosto de 2018, todos los sitios web ecuatorianos que presten un servicio público deben ser accesibles WCAG 2.0 nivel A.

- Transitoria Segunda. Los propietarios de los sitios web a los que se aplica este reglamento técnico tendrán un plazo de 4 años para adecuar sus sitios web existentes al momento de entrar en vigencia el reglamento de acuerdo al nivel de conformidad AA de la norma INEN vigente. El 8 de agosto de 2020, todos los sitios web ecuatorianos que presten un servicio público deben ser accesibles WCAG 2.0 nivel AA.

\section{Herramientas de evaluación de accesibilidad de los sitios web.}

Existen diferentes sistemas para comprobar si una página Web es accesible, entre ellos cabe reseñar una serie de herramientas automatizadas que nos ayudan a evaluar, a través de la verificación de los estándares de facto, la accesibilidad global que presentan los contenidos de un portal Web y determinar cuáles son los puntos fuertes y débiles susceptibles de mejora (Mascaraque, 2009). La W3C las define a estas herramientas como programas de software o servicios en línea que ayudan a determinar si el contenido web cumple con las pautas de accesibilidad (World Wide Web Consortium, 2016). Una herramienta de este tipo nunca puede sustituir la revisión que realiza un experto en accesibilidad web, por lo que se deben emplear como un primer paso, pero no el único (Luján-Mora, 2019b).

AccessMonitor. Es un validador automático que comprueba la aplicación de las directrices de accesibilidad en el contenido HTML de una página web, utilizando las directrices de la WCAG 2.0 de la W3C, con una batería de 86 pruebas (AccessMonitor, 2012).

Test de accesibilidad web (TAW). Es un programa de evaluación automática desarrollada por la fundación CTIC, sirve para analizar la accesibilidad de los sitios web de acuerdo a las tres prioridades de la WCAG 2.0 del W3C (TAW, 2019).

Observatorio de Accesibilidad Web (OAW). Es un analizador en línea elaborado por el centro de Investigación Desarrollo e Innovación en Ingenierías con los Laboratorios de: Tecnologías de Inclusión e Inteligencia Artificial de la Universidad Politécnica Salesiana. Esta herramienta

\footnotetext{
\begin{tabular}{l|l}
220 & Vol. 5, núm. 3, julio, 2019, pp.212-237 \\
Diego German Salas Paucar, Milton Alfredo Campoverde Molina
\end{tabular}
} 
permite el monitoreo del cumplimiento de la normativa ISO/IEC 40500, por medio de su API REST. Imprime los aciertos y posibles errores cometidos en el desarrollo de la página web, mediante la verificación de los criterios de éxito asociados a los cumplimientos del estándar WCAG 2.0 .

Web accessibility evaluation tool (WAVE). Es un analizador en línea utilizado para evaluar la accesibilidad de páginas web. Perite evaluar bajo las pautas de accesibilidad de WCAG 2.0 en los niveles A y AA, no admite AAA (WebAIM, 2019).

Markup Validation Service. Es una herramienta en línea de la W3C gratuita que ayuda a verificar la validez de los documentos web escritos en lenguajes de marcado HTML o XHTML.

CSS Validation Service. Es una herramienta creada por la W3C creada para validar hojas de estilo de cascada (CSS), es muy útil para las personas que escriben CSS ya que les permite comprobar y corregir sus hojas de estilo de cascada.

What CMS. Es una herramienta web que permite verificar si una página está haciendo uso de un gestor de contenidos CMS o no (WhatCMS, 2019).

\section{Trabajos relacionados}

En el año 2019 se realizó un análisis de accesibilidad de 191 web de las instituciones educativas en la ciudad de Cuenca, Ecuador. El propósito de la investigación fue evaluar la accesibilidad de los portales web con las WCAG 2.0 con un nivel de conformidad A, utilizando las herramientas en línea Examinator, TAW, Markup Validation Service y CSS Validation Service. Se concluye que se requiere corregir errores en todos los portales web de las instituciones educativas de la ciudad de Cuenca analizadas. Además, se concluye que, por el promedio de errores encontrados, es más factible que las instituciones educativas rediseñen sus portales web, a fin de cumplir con las WCAG 2.0. También pueden mejorar el cumplimiento de las WCAG 2.0, con un arduo trabajo de revisión y corrección del código fuente de sus portales web (Campoverde \& Valverde, 2019).

En el año 2019, una investigación se realizó la evaluación de la accesibilidad en el sitio web de la Universidad Península de Santa Elena. Se ha realizado comprobando el cumplimiento de las pautas

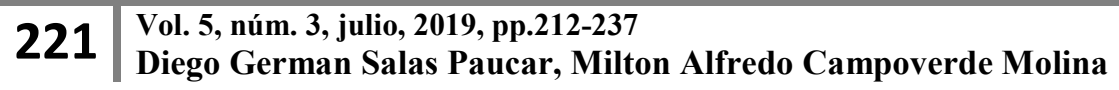


de accesibilidad de contenidos Web establecidas por el W3C en WCAG 2.0. Mediante la realización de tres valoraciones diferentes como una evaluación de accesibilidad automática utilizando la herramienta de tasación en línea TAW, como método complementario una revisión manual acudiendo a softwares específicos para dicha tarea, además se añadieron test presenciales con usuarios que poseen distintos niveles y tipos de discapacidad visual. Determinaron que el portal web no cumple con un mínimo de cumplimiento de las normas INEN -ISO/IEC 40500 por lo tanto concluyeron que no es accesible y se recomiendo a dicha institución tomar las medidas necesarias para cumplir con las pautas de accesibilidad web y el reglamento INEN -ISO/IEC 40500 (Luis Rodríguez V, 2019).

En el año 2018 se realizó un estudio referente al cumplimiento de los estándares establecidos por el $\mathrm{W} 3 \mathrm{C}$ respecto de la accesibilidad web. Se aplicaron dos validadores a las páginas web pertenecientes a un portal educativo estatal. Las validaciones las realizaron aplicando las herramientas TAW y EvalAccess 2.0 a cinco páginas representativas del portal. Los resultados, se analizaron considerando las WCAG 2.0 establecidas por el W3C. Al utilizar TAW en la validación de las páginas, se obtuvieron errores que en su mayoría corresponden al nivel de conformidad A y aplicando EvalAccess 2.0, se detectaron fallas relacionadas a la prioridad 2, imposibilitando el logro del nivel de conformidad AA, y más grave aún, se registraron falencias referidas a las pautas de la prioridad 1, impidiendo el cumplimento del nivel de conformidad A. Por lo tanto, con ambas herramientas se coincide en que ninguna de las páginas evaluadas logra alcanzar el nivel de conformidad A (Pagnoni \& Mariño, 2018).

En el año 2019 se realizó el análisis retrospectivo de la accesibilidad de los portales educativos en Ecuador. La investigación presenta una comparación entre 2016 y 2018 del nivel de accesibilidad de las páginas web educativas. Para el análisis utilizaron la herramienta en línea TAW, mediante esta herramienta pudieron evaluar el cumplimiento de HTML y CSS con WCAG 2.0. Seleccionaron 31 sitios web que manejan el dominio.edu.ec y determinaron las páginas web a las que los estudiantes acceden con mayor frecuencia. El análisis se hizo con un nivel de conformidad “A" en 2016 y 2018. Se concluyó que los sitios web no son lo suficiente robustos en ninguno de los dos años en los que se hizo el análisis, además que es necesario corregir errores en todos los

\footnotetext{
\begin{tabular}{|l|l|}
222 & Vol. 5, núm. 3, julio, 2019, pp.212-237 \\
Diego German Salas Paucar, Milton Alfredo Campoverde Molina
\end{tabular}
} 
portales web analizados. Debido al nivel y la cantidad de errores reportados, algunas unidades educativas y colegios encuentran más factible rediseñar sus portales web para cumplir con las pautas de accesibilidad web, y estar acorde a los tiempos establecidos en el reglamento técnico ecuatoriano RTE INEN 288 para el nivel de conformidad “AA” en el 2020 (Campoverde Molina, Luján-Mora, \& Valverde, 2019).

\section{Metodología}

La metodología a adoptar en esta investigación se basa en las directrices y pautas que permiten la realización exitosa de esta investigación. A continuación, se detalla los pasos que se ha seguido para la realización de este proyecto.

- Selección de portales web empresariales según el dominio.com y dominio.com.ec: para lo cual se recurre a la información que consta en las bases de datos de superintendencia de compañías valores y seguros (Superintendencia de Compañías Valores y Seguros, 2019). Analizando la base de datos de las empresas activas en la provincia de Loja se determinó que existen 500 compañías activas de las cuales 46 poseen portales web que manejan el dominio.com y dominio.com.ec.

- $\quad$ Selección de páginas web: la de cada uno de los portales web se seleccionaron 4 páginas web de muestra y los que tienen menos de 4 todas sus páginas web.

- Evaluación de las páginas de inicio de los portales web: estas páginas permiten a los usuarios desplazarse entre los demás sitios de los portales web. Para realizar la evaluación de las 46 páginas de inicio de los portales web se utilizaron herramientas en línea como; AccesMonitor y el Observatorio de accesibilidad web OAW, esto con el fin de determinar el porcentaje de cumplimiento del nivel de conformidad AA de la WCAG 2.0. Además, se realizó el análisis para determinar que páginas web empresariales estaban diseñadas con un gestor de contenidos.

- $\quad$ Evaluación de las páginas seleccionadas de los portales web: para determinar los errores de accesibilidad en las 46 páginas de inicio y las 138 páginas seleccionadas, se utilizaron herramientas en línea como; TAW, Web Accessibility Evaluation Tool WAVE, Markup Validation Service, y CSS Validation Service.

\footnotetext{
\begin{tabular}{|l|l}
223 & Vol. 5, núm. 3, julio, 2019, pp.212-237 \\
Diego German Salas Paucar, Milton Alfredo Campoverde Molina
\end{tabular}
} 
- Nivel de cumplimiento y porcentaje de cumplimiento: para establecer el nivel de cumplimiento se verificó los iconos que certifiquen la accesibilidad de los sitios por la W3C en HTML, CSS y sus niveles de conformidad A, AA y AAA. A partir de los datos obtenidos de la evaluación de las herramientas automáticas se corrobora el nivel de cumplimiento de los criterios de éxito de la WCAG 2.0 con un nivel de conformidad AA.

\section{Resultados}

\section{Selección de portales web según el dominio.com y dominio.com.ec}

En la página web de la superintendencia de compañías (Superintendencia de Compañías Valores y Seguros, 2019) consta el listado de las compañías creadas en el Ecuador, clasificadas por región y provincia, además las que se encuentran activas e inactivas. Para este análisis se ha seleccionado los portales web de 46 empresas con el dominio.com y domino.com.ec. de las 500 empresas activas en la provincia de Loja en el 2019. En la tabla 2 se detalla la abreviatura utilizada, el nombre de cada compañía y la URL del portal web.

Tabla 2. Portales Web seleccionados con el dominio.com y dominio.com.ec.

\begin{tabular}{|c|c|c|}
\hline Abreviatura & Nombre de la compañía & $\begin{array}{l}\text { URL } \quad \text { Web } \\
\text { (.com/.com.ec) }\end{array}$ \\
\hline adeplan & Adeplan Cia. Ltda. & http://www.adeplan.com \\
\hline agemic & Agemic Cia.Ltda. & http://consultora-agemic.com \\
\hline aguamania & Aguamania Cia. Ltda. & http://www.aguamania.com.ec \\
\hline arcimego & Arcimego c..a. & http://www.arcimego.com \\
\hline atlantis & Atlantis travel fec CÍa. Ltda & $\begin{array}{l}\text { http://www.atlantistravel.com. } \\
\text { ec }\end{array}$ \\
\hline bratya & Bratya-azanza Cia.Ltda. & http://bratya-azanza.com \\
\hline cafrilosa & Cafrilosa Loja S.A. & http://www.cafrilosa.com \\
\hline cevascop & Cevascop S.A. & http://www.medilab.com.ec \\
\hline conducir & Cenforcaper CÍa. Ltda. & http://www.conducirloja.com \\
\hline cronica & Crónica de la tarde CÍa. Ltda. & http://www.cronica.com.ec \\
\hline cuadricar & Cuadricar rent a car CÍa. Ltda & $\begin{array}{l}\text { http://www.cuadricarentacar.c } \\
\text { om }\end{array}$ \\
\hline decorteja & $\begin{array}{l}\text { Empresa de tejas Decorteja CÍa. } \\
\text { Ltda }\end{array}$ & $\overline{\mathrm{http}}: / /$ decorteja.com \\
\hline delaromas & Delaroma S. A. & http://www.delaroma.com.ec \\
\hline $\begin{array}{l}\text { distrigrand } \\
\text { a }\end{array}$ & $\begin{array}{l}\text { Distribuidora Granda distrigranda } \\
\text { CÍla. Ltda. }\end{array}$ & http://distribuidoragranda.com \\
\hline
\end{tabular}


Dom. Cien., ISSN: 2477-8818

Vol. 5, núm. 3, julio, 2019, pp. 212-237

Accesibilidad en los sitios web empresariales de la provincia de Loja. Análisis preliminar

\begin{tabular}{|c|c|c|}
\hline ec\&t & Constructora ec\&t CÍa. Ltda & $\begin{array}{l}\text { http://www.constructoraect.co } \\
\underline{\mathrm{m}}\end{array}$ \\
\hline ecomaxi & Autoservicio ecomaxi-jc CÍa. Ltda. & http://ecomaxi.com.ec \\
\hline edihora & $\begin{array}{l}\text { Editorial la hora de Loja edihora } \\
\text { CÍa. Ltda. }\end{array}$ & http://lahora.com.ec \\
\hline educaes & $\begin{array}{l}\text { Centro de formación y capacitación } \\
\text { Educaes CÍa. Ltda. }\end{array}$ & http://centroeducaes.com \\
\hline elsur & $\begin{array}{l}\text { Comercial el sur comercial Elsur } \\
\text { s.a. }\end{array}$ & http://elsursa.com \\
\hline farb & Farb constructores CÍa. Ltda. & http://constructorafarb.com \\
\hline hve & $\begin{array}{l}\text { Hotelera Valdivieso \& Eguiguren } \\
\text { CÍ́a. Ltda. }\end{array}$ & $\begin{array}{l}\text { http://www.grandvictoriabh.c } \\
\text { om }\end{array}$ \\
\hline ile & $\begin{array}{l}\text { Industria lojana de especerias ile } \\
\text { C.A. }\end{array}$ & http://ile.com.ec \\
\hline ilelsa & $\begin{array}{l}\text { Industria licorera embotelladora de } \\
\text { loja S.A }\end{array}$ & http://www.ilelsa.com \\
\hline iomotors & Iomotors S.A. & http://www.iomotors.com.ec \\
\hline kradac & Kradac CÍa. Ltda. & http://kradac.com \\
\hline lasureñita & La sureñita S.A. & http://lasurenita.com.ec \\
\hline lavacor & Importadora Lavacor CÍa. Ltda. & $\begin{array}{l}\text { http://www.importadoralavac } \\
\text { or.ec }\end{array}$ \\
\hline lojacasa & Cobijosloja CÍa. Ltda. & http://www.lojacasa.com.ec \\
\hline lojafar & Distribuidora Lojafar C.A. & http://lojafarca.com \\
\hline lojagas & $\begin{array}{l}\text { Compañía de economía mixta } \\
\text { Lojagas }\end{array}$ & http://lojagas.com.ec \\
\hline lojare & Importadora lojare CÍa. Ltda. & http://importadoralojare.com \\
\hline lojasalud & Loja salud CÍa. Ltda. & http://lojasalud.com \\
\hline malca & Monterrey azucarera lojana Malca & http://www.malca.ec \\
\hline mfc & $\begin{array}{l}\text { Mercantiles Flores Campoverde } \\
\text { CÍa. Ltda. }\end{array}$ & $\begin{array}{l}\text { http://mercantilflorescampove } \\
\text { rde.com }\end{array}$ \\
\hline minasur & Importadora minasur CÍa. Ltda. & http://minasur.com \\
\hline nodo & Electritelecom CÍa. Ltda. & http://www.nodo.com.ec \\
\hline nsim & Nsim CÍa. Ltda. & http://nsim.com.ec \\
\hline ortega & Importadora Ortega CÍa. Ltda. & $\begin{array}{l}\text { http://www.importadoraortega } \\
\text {.com }\end{array}$ \\
\hline quipus & Quipus CÍa. Ltda. & http://quipus.com.ec \\
\hline radiopoder & Radio poder CÍa. Ltda. & http://radiopoderfm.com \\
\hline segurpi & Segurpi CÍa. Ltda. & http://segurpiseguridad.com \\
\hline sistemas & Serviestudios CÍa. Ltda. & http://www.sistemas.com.ec \\
\hline solgas & Paneles solares solgas S. A. & http://solgas.lojagas.com \\
\hline superlaser & $\begin{array}{l}\text { Servicios de radiodifusión Ojeda } \\
\text { Valarezo CÍa. Ltda. }\end{array}$ & http://superlaserfm.com.ec \\
\hline
\end{tabular}




\begin{tabular}{|l|l|l|}
\hline surectel & $\begin{array}{l}\text { Surectel ingeniería y proyectos } \\
\text { CÍla. Ltda. }\end{array}$ & $\mathrm{http:// \text {www.surectel.com.ec }}$ \\
\hline $\begin{array}{l}\text { vilcainterag } \\
\text { uas }\end{array}$ & $\begin{array}{l}\text { Vilcabamba internacional de aguas } \\
\text { CÍa. Ltda. }\end{array}$ & $\begin{array}{l}\text { http://www.aguadevilcabamba } \\
\text {.com.ec }\end{array}$ \\
\hline
\end{tabular}

Fuente: Elaboración propia

\section{Selección de páginas web}

Los portales web disponen de un número significativo de páginas web, para esta investigación se seleccionaron como muestra 4 páginas web por cada portal web de las compañías. En la Tabla 3 se puede ver las empresas y las páginas seleccionadas por cada portal web.

Tabla 3. Páginas web seleccionadas del portal web de cada compañía.

\begin{tabular}{|c|c|c|}
\hline $\begin{array}{c}\mathbf{N} \\
0\end{array}$ & Empresas & Páginas seleccionadas en los portales web \\
\hline 1 & adeplan & Página de inicio, servicios, clientes, personal \\
\hline 2 & agemic & Página de inicio, acerca, proyectos, contactos \\
\hline 3 & aguamania & Página de inicio, misión y visión, reglamento, normas \\
\hline 4 & arcimego & Página de inicio, empresa, productos, noticias \\
\hline 5 & atlantis & Página de inicio, servicios, galería, tours \\
\hline 6 & bratya & Página de inicio, servicios, leyes publicadas, sobre nosotros \\
\hline 7 & cafrilosa & Página de inicio, nosotros, nuestros productos, novedades \\
\hline 8 & cevascop & Página de inicio, menú, laboratorio, imágenes \\
\hline 9 & conducir & Página de inicio, nosotros, cursos, contacto \\
\hline 10 & crónica & $\begin{array}{l}\text { Página de inicio, quienes somos, información, } \\
\text { entretenimiento }\end{array}$ \\
\hline 11 & cuadricar & Página de inicio, quienes somos, servicios, contacto \\
\hline 12 & decorteja & Página de inicio, contacto, nosotros, distribuidores \\
\hline 13 & delaromas & Página de inicio, quienes somos, productos, contactos \\
\hline 14 & $\begin{array}{l}\text { distrigrand } \\
\text { a }\end{array}$ & $\begin{array}{l}\text { Página de inicio, misión y visión, nuestros proveedores, } \\
\text { contacto }\end{array}$ \\
\hline 15 & ec\&t & Página de inicio, quienes somos, contacto, servicios \\
\hline 16 & ecomaxi & $\begin{array}{l}\text { Página de inicio, consulta tu factura, quienes somos, } \\
\text { contacto }\end{array}$ \\
\hline 17 & edihora & Página de inicio, noticias, opinión, secciones \\
\hline 18 & educaes & Página de inicio, quienes somos, servicios, contacto \\
\hline 19 & elsur & Página de inicio, servicios, empresa, contacto \\
\hline 20 & farb & Página de inicio, contacto servicios nosotros \\
\hline 21 & hve & Página de inicio, habitaciones, restaurantes, servicios \\
\hline 22 & ile & Página de inicio, contacto, noticias, productos \\
\hline
\end{tabular}




\begin{tabular}{|l|l|l|}
\hline 23 & ilelsa & Página de inicio, productos, contacto, somos ilelsa \\
\hline 24 & iomotors & Página de inicio, contacto, postventa, io motors \\
\hline 25 & kradac & $\begin{array}{l}\text { Página de inicio, productos y servicios monitoreo contacto } \\
\text { Página de inicio, acerca de nosotros, catálogo de productos, } \\
\text { contactos }\end{array}$ \\
\hline 26 & lasureñita & Página de inicio, quienes somos, productos, contactos \\
\hline 27 & lavacor & Página de inicio, servicios, clientes, contacto \\
\hline 28 & lojacasa & Página de inicio, quienes somos, líneas, noticias \\
\hline 29 & lojafar & Página de inicio, registro, términos y condiciones, políticas \\
\hline 30 & lojagas & Página de inicio, marcas, nosotros, contacto \\
\hline 31 & lojare & Página de inicio, especialidades, infórmate, contacto \\
\hline 32 & lojasalud & Página de inicio, productos, sostenibilidad, servicio al cliente \\
\hline 33 & malca & Página de inicio, quienes somos, contacto, tienda \\
\hline 34 & mfc & Página de inicio, quienes somos, contacto, premiaciones \\
\hline 35 & minasur & Página de inicio, quienes somos, certificaciones, contacto \\
\hline 36 & nodo & Página de inicio, académico, utilitario, contacto \\
\hline 37 & nsim & Página de inicio, tienda, compras, contacto \\
\hline 38 & ortega & Página de inicio, quienes somos, servicios, noticias \\
\hline 39 & quipus & Página de inicio, entrevistas, programación, contacto \\
\hline 40 & radiopoder & Página de inicio, contacto, servicios, nosotros \\
\hline 41 & segurpi & Página de inicio, quienes somos, contacto, académico \\
\hline 42 & sistemas & Página de inicio, nosotros, productos, servicios \\
\hline 43 & solgas & Página de inicio, quienes somos, programación, contacto \\
\hline 44 & superlaser & Página de inicio, quienes somos, servicios, contacto \\
\hline 45 & surectel & $\begin{array}{l}\text { Página de inicio, corrientes subterán, } \\
\text { contacto }\end{array}$ \\
\hline 46 & vilcaintera \\
\hline
\end{tabular}

Fuente: Elaboración propia

\section{Evaluación de las páginas de inicio de los portales web}

Para la evaluación de las páginas de inicio se utilizó dos herramientas; OAW y AccessMonitor con ellas se verifico si las páginas cumplen con las WCAG 2.0 y un nivel de conformidad AA. La herramienta de la Universidad Politécnica Salesiana OAW evalúa 70 criterios de éxito asociados al cumplimiento "AA" del estándar WCAG 2.0. Para el análisis se seleccionaron los siguientes parámetros; Resolución de pantalla por defecto 1366x768, nivel de conformidad AA y WCAG 2.0. La herramienta web AccessMonitor permite cuantificar en una escala del 1 al 10 el nivel de accesibilidad alcanzado por una página web.

En la figura 1 se muestra el resultado obtenido con el OAW en el principio perceptible (P) en el cual solo el 4\% de las páginas web cumplen el nivel de conformidad AA de la WCAG 2.0.

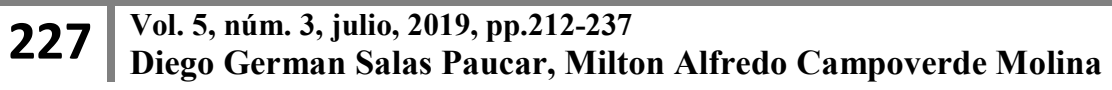


Dom. Cien., ISSN: 2477-8818

Vol. 5, núm. 3, julio, 2019, pp. 212-237

Accesibilidad en los sitios web empresariales de la provincia de Loja. Análisis preliminar

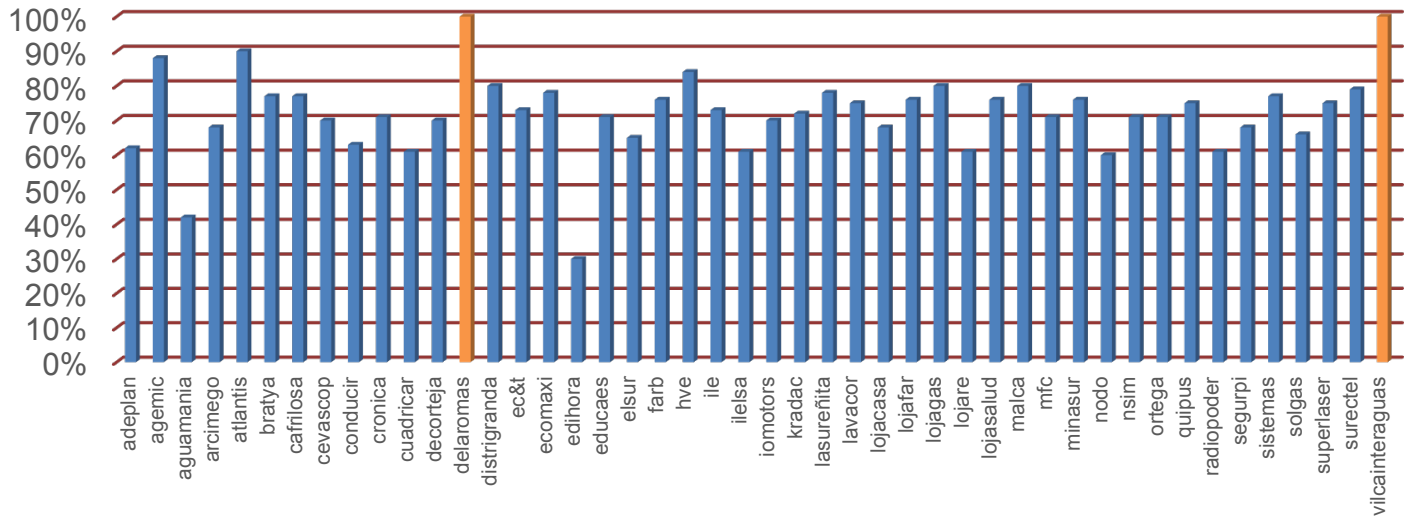

Figura 1. Porcentaje de cumplimiento del principio Perceptible Fuente: Elaboración propia

En la figura 2 se muestra el resultado obtenido con el OAW en el principio operable $(\mathrm{O})$ un $25 \%$ de las páginas cumplen el nivel de conformidad AA de la WCAG 2.0.

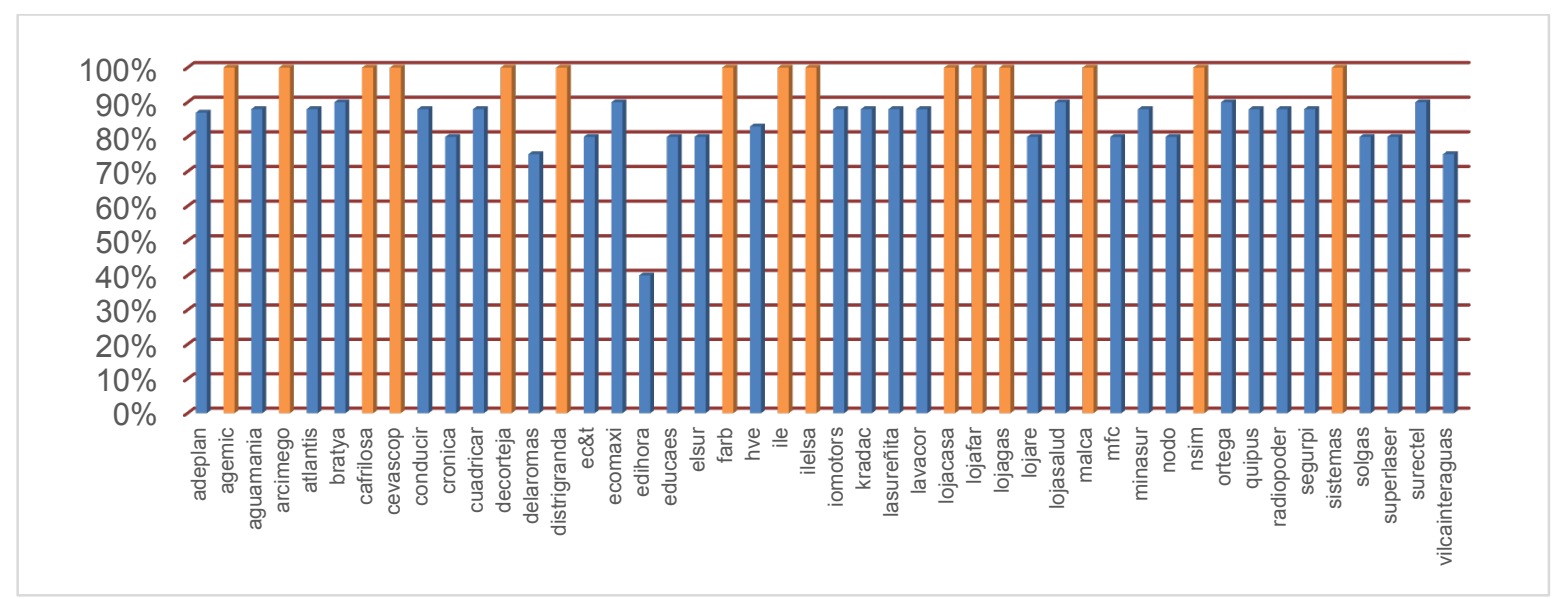

Figura 2. Porcentaje de cumplimiento del principio Operable

Fuente: Elaboración propia

En la figura 3 se muestra el resultado obtenido con el OAW en el principio comprensible (C) un $18 \%$ cumple con el nivel de conformidad AA de la WCAG 2.0. 
Dom. Cien., ISSN: 2477-8818

Vol. 5, núm. 3, julio, 2019, pp. 212-237

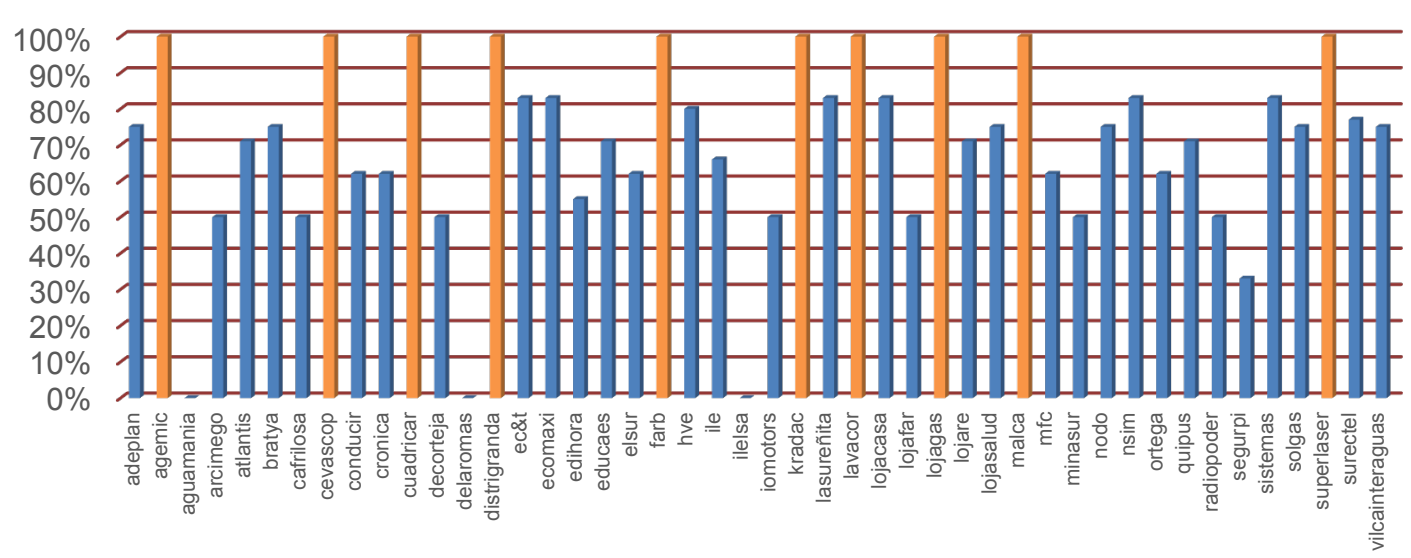

Figura 3. Porcentaje de cumplimiento del principio Comprensible Fuente: Elaboración propia

En la figura 4 se muestra el resultado obtenido con el OAW en el principio robusto $(\mathrm{R})$ un $6 \%$ de las páginas web cumple el nivel de conformidad AA de la WCAG 2.0.

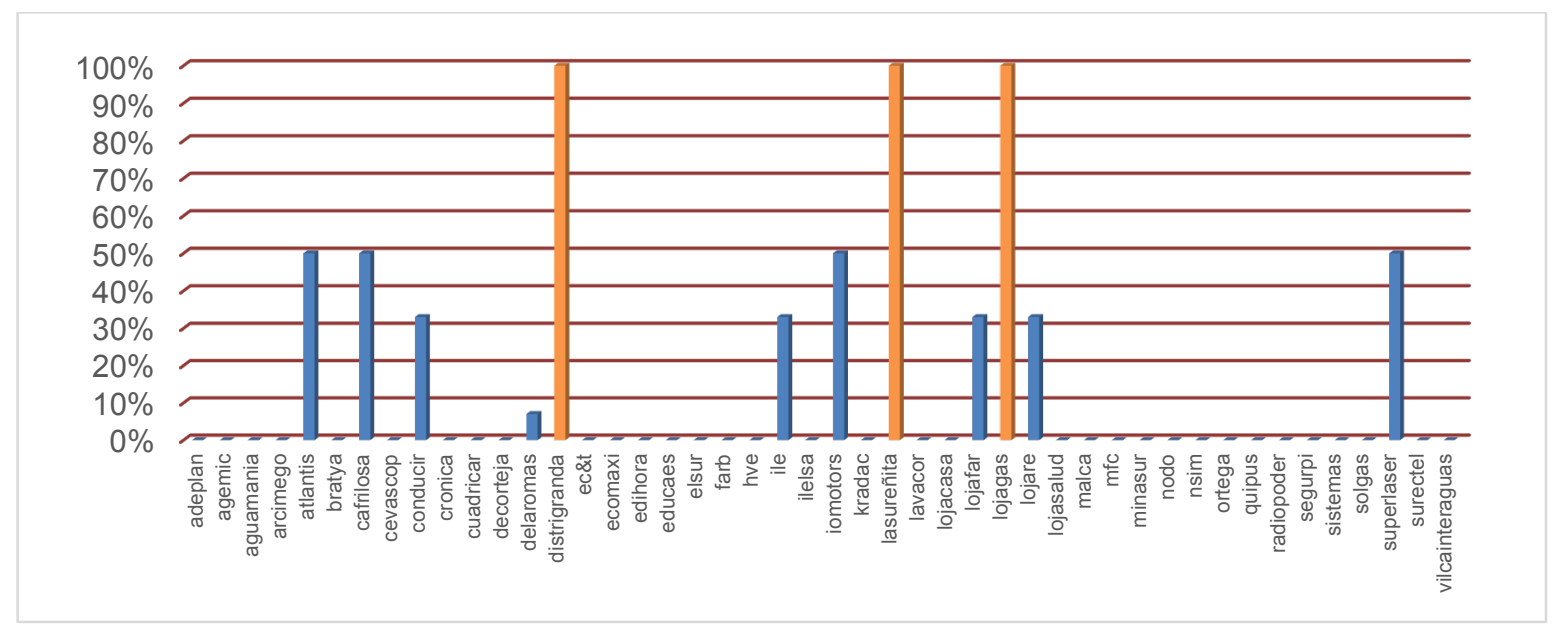

Figura 4. Porcentaje de cumplimiento del principio Robusto Fuente: Elaboración propia

En el análisis realizado en AccessMonitor se seleccionó WCAG 2.0 y se obtuvo un promedio de 5.94 en accesibilidad en las páginas web. En la figura 5 se muestra en orden descendente los valores porcentuales de los datos de accesibilidad obtenidos con AccessMonitor. 


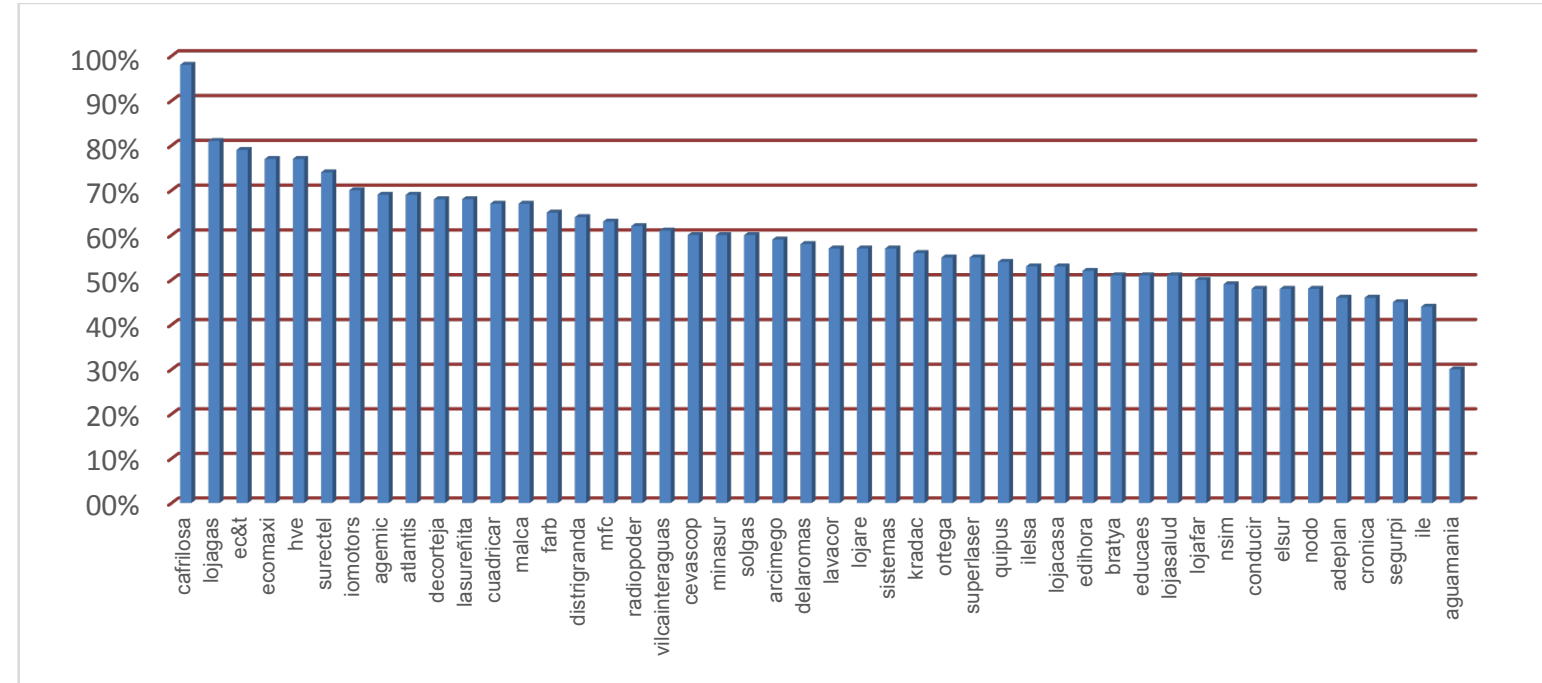

Figura 5. Porcentaje de accesibilidad Access Monitor Fuente: Elaboración propia

Se realizó un análisis de los portales web principales para determinar cuántos usan gestores de contenidos y se determinó que 34 portales sí disponen de CMS, de los cuales 17 usan WordPress, 15 Joomla, 1 Drupal y 1 Adobe Muse, 12 paginas no disponen de CMS.

\section{Evaluación de las páginas de los portales web.}

Para el análisis de cada una de las páginas de los portales web seleccionados, se utilizó las siguientes herramientas en line; TAW que permitió determinar los errores por cada uno de sus principios: perceptible $(\mathrm{P})$, operable $(\mathrm{O})$, comprensible $(\mathrm{C})$ y robusto $(\mathrm{R})$; WAVE analiza los problemas de cumplimiento de accesibilidad que se encuentran en las pautas de la Sección 508 y WCAG 2.1 (WebAIM, 2019); Markup Validation Service de la W3C, para evaluar el lenguaje de marcado (HTML) y determinar los errores y CSS Validation Service de la W3C para validar Hojas de Estilo en Cascada (CSS), y determinar errores comunes, tipográficos y usos indebidos de CSS.

En la tabla 4 se muestra los resultados obtenidos por la evaluación del contenido HTML con Markup Validation Service y CSS con CSS Validation Service. 
Tabla 4. Resultados porcentuales del análisis de las páginas web con Markup Validation Service HTML y CSS Validation Service.

\begin{tabular}{|l|l|l|}
\hline Herramienta de & $\begin{array}{l}\text { Porcentaje de } \\
\text { errores }\end{array}$ & $\begin{array}{l}\text { Porcentaje } \\
\text { advertencias }\end{array}$ \\
\hline $\begin{array}{l}\text { HTML Markup Validation } \\
\text { Service }\end{array}$ & $40 \%$ & $60 \%$ \\
\hline CSS Validation Service & $1 \%$ & $99 \%$ \\
\hline
\end{tabular}

Fuente: Elaboración propia

En la tabla 5 se muestra los errores en las 184 páginas analizadas de los 46 portales web correspondientes a cada empresa. El analizador TAW presenta los errores según los principios perceptibles $(\mathrm{P})$, operable $(\mathrm{O})$, comprensible $(\mathrm{C})$ y robusto $(\mathrm{R})$. El analizador web WAVE presenta los errores de accesibilidad y errores de contaste. Además, se presenta los errores obtenidos al analizar el contenido HTML y CSS.

Tabla 5. Total, de errores en las páginas seleccionadas de los portales web, según cada validador.

\begin{tabular}{|c|c|c|c|c|c|c|c|c|c|}
\hline \multirow{2}{*}{ 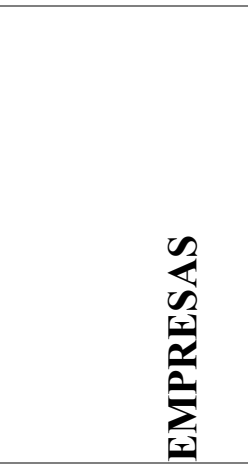 } & \multirow{2}{*}{ 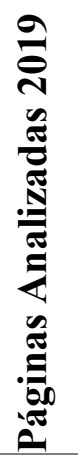 } & \multicolumn{4}{|c|}{ 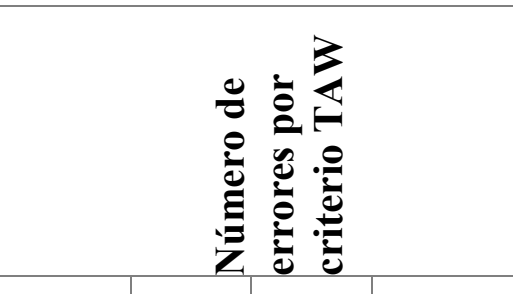 } & \multirow{2}{*}{ 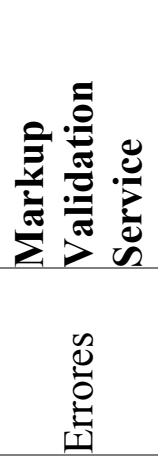 } & \multirow{2}{*}{ 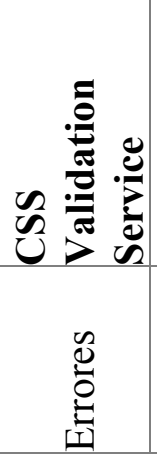 } & $\begin{array}{l}\stackrel{0}{0} \\
\stackrel{0}{0} \\
\stackrel{0}{\Xi}\end{array}$ & \multirow{2}{*}{ 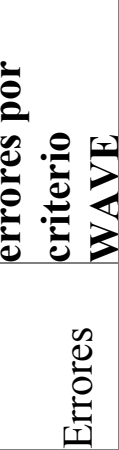 } \\
\hline & & $a$ & 0 & $U$ & $\simeq$ & & & 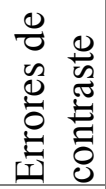 & \\
\hline Adeplan & 4 & 336 & 149 & 76 & 72 & 17 & 1 & 0 & 24 \\
\hline Agemic & 4 & 783 & 467 & 76 & 23 & 2 & 71 & 0 & 169 \\
\hline Aguamania & 4 & 1155 & 345 & 66 & 306 & 43 & 4 & 18 & 47 \\
\hline Arcimego & 4 & 241 & 160 & 29 & 611 & 33 & 40 & 108 & 29 \\
\hline Atlantis & 4 & 105 & 144 & 92 & 190 & 8 & 1 & 59 & 19 \\
\hline Bratya & 4 & 571 & 156 & 85 & 196 & 10 & 2 & 96 & 42 \\
\hline Cafrilosa & 4 & 1196 & 157 & 56 & 146 & 6 & 2 & 18 & 17 \\
\hline Cevascop & 4 & 200 & 139 & 20 & 30 & 12 & 16 & 3 & 41 \\
\hline Conducer & 4 & 48 & 68 & 30 & 36 & 12 & 50 & 34 & 14 \\
\hline Cronica & 4 & 407 & 278 & 80 & 276 & 63 & 21 & 290 & 108 \\
\hline Cuadricar & 4 & 151 & 90 & 56 & 50 & 5 & 6 & 77 & 3 \\
\hline Decorteja & 4 & 323 & 107 & 76 & 160 & 2 & 12 & 32 & 28 \\
\hline Delaromas & 4 & 118 & 102 & 39 & 68 & 10 & 32 & 25 & 26 \\
\hline Distrigranda & 4 & 679 & 365 & 30 & 95 & 72 & 4 & 18 & 295 \\
\hline
\end{tabular}

231 Vol. 5, núm. 3, julio, 2019, pp.212-237

Diego German Salas Paucar, Milton Alfredo Campoverde Molina 


\begin{tabular}{|l|r|r|r|r|r|r|r|r|r|}
\hline ec\&t & 4 & 85 & 84 & 20 & 16 & 2 & 16 & 31 & 8 \\
\hline Ecomaxi & 4 & 305 & 66 & 34 & 104 & 3 & 12 & 14 & 15 \\
\hline Edihora & 4 & 1260 & 1213 & 28 & 413 & 141 & 9 & 170 & 437 \\
\hline Educaes & 4 & 62 & 116 & 88 & 52 & 5 & 15 & 63 & 17 \\
\hline Elsur & 4 & 210 & 98 & 91 & 611 & 5 & 15 & 57 & 35 \\
\hline Farb & 4 & 121 & 97 & 38 & 210 & 8 & 5 & 2 & 21 \\
\hline Hve & 4 & 53 & 97 & 24 & 421 & 0 & 3 & 0 & 16 \\
\hline Ile & 4 & 568 & 319 & 88 & 61 & 17 & 35 & 121 & 203 \\
\hline Ilelsa & 4 & 127 & 137 & 30 & 193 & 51 & 7 & 3 & 24 \\
\hline Iomotors & 4 & 179 & 109 & 40 & 94 & 18 & 20 & 17 & 26 \\
\hline Kradac & 4 & 220 & 112 & 35 & 123 & 6 & 14 & 40 & 16 \\
\hline Lasureñita & 4 & 128 & 80 & 34 & 27 & 2 & 13 & 27 & 12 \\
\hline Lavacor & 4 & 219 & 98 & 27 & 188 & 4 & 0 & 78 & 34 \\
\hline Lojacasa & 4 & 416 & 103 & 48 & 256 & 41 & 44 & 17 & 71 \\
\hline Lojafar & 4 & 193 & 89 & 88 & 22 & 6 & 0 & 55 & 17 \\
\hline Lojagas & 4 & 396 & 95 & 78 & 38 & 0 & 2 & 0 & 0 \\
\hline Lojare & 4 & 343 & 201 & 76 & 83 & 0 & 9 & 51 & 4 \\
\hline Lojasalud & 4 & 83 & 103 & 59 & 26 & 2 & 3 & 94 & 36 \\
\hline Malca & 4 & 68 & 92 & 21 & 2925 & 4 & 8 & 45 & 11 \\
\hline Mfc & 4 & 177 & 186 & 57 & 403 & 23 & 12 & 69 & 30 \\
\hline Minasur & 4 & 199 & 64 & 13 & 25 & 2 & 8 & 36 & 8 \\
\hline Nodo & 4 & 547 & 81 & 58 & 1638 & 9 & 36 & 55 & 22 \\
\hline Nsim & 4 & 169 & 98 & 41 & 485 & 23 & 12 & 25 & 40 \\
\hline Ortega & 4 & 576 & 429 & 142 & 1921 & 8 & 28 & 285 & 74 \\
\hline Quipus & 4 & 339 & 129 & 158 & 47 & 2 & 13 & 10 & 44 \\
\hline Radiopoder & 4 & 1117 & 103 & 54 & 482 & 4 & 5 & 43 & 269 \\
\hline Segurpi & 4 & 93 & 157 & 95 & 74 & 8 & 5 & 11 & 19 \\
\hline Sistemas & 4 & 292 & 124 & 26 & 3260 & 3 & 4 & 1 & 11 \\
\hline Solgas & 4 & 279 & 110 & 56 & 146 & 5 & 8 & 1 & 25 \\
\hline Superlaser & 4 & 196 & 102 & 21 & 3014 & 8 & 121 & 136 & 33 \\
\hline Surectel & 4 & 89 & 60 & 76 & 23 & 7 & 1 & 42 & 8 \\
\hline vilcainteraguas & 4 & 157 & 113 & 34 & 169 & 3 & 2 & 25 & 20 \\
\hline \multicolumn{1}{|c|}{ TOTAL: } & $\mathbf{1 8 4}$ & $\mathbf{1 5 5 7 9}$ & $\mathbf{7 7 9 2}$ & $\mathbf{2 5 8 9}$ & $\mathbf{1 9 8 0 9}$ & $\mathbf{7 1 2}$ & $\mathbf{7 4 2}$ & $\mathbf{2 4 0 2}$ & $\mathbf{2 4 6 8}$ \\
\hline & & & & & & & \\
\hline
\end{tabular}

Fuente: Elaboración propia

Con la evaluación de accesibilidad de las páginas web mediante TAW se obtuvo los siguientes resultados; $16 \%$ son problemas, $78 \%$ son advertencias y $6 \%$ corresponde a No Verificado. Con la evaluación de WAVE se obtuvo los siguientes resultados; $17 \%$ errores, $21 \%$ alertas, $14 \%$ características, $21 \%$ elementos estructurales, 10\% HTML5 y ARIA y $17 \%$ Errores de contraste. 
Para cumplir con los principios de accesibilidad web de WCAG 2.0 y sus niveles de conformidad, se debe corregir los errores que se presentan en los principios analizados por TAW. Los errores que se presentan con el analizador WAVE y los errores de los lenguajes de marcado HTML o XHTML y las hojas de estilo en cascada CSS.

\section{Nivel de cumplimiento}

Una vez realizado en análisis de accesibilidad de las páginas de los portales web, se pudo constatar que no poseen los iconos de la W3C que certifican la accesibilidad en CSS, HTML o los niveles de conformidad A, AA, AAA. En el análisis realizado con la herramienta OAW se pudo verificar que ninguna página de inicio de los portales web alcanza el 100\% en cumplimiento en todos los principios analizados. El principio robusto es el que presenta el menor porcentaje de cumplimiento de los 4 analizados, seguido por el perceptible. Con la herramienta TAW se pudo comprobar que los principios que mayor número de errores presentan son el robusto seguido por el perceptible. Se debe corregir los errores encontrados en las páginas web para así cumplir con el reglamento técnico ecuatoriano RTE INEN 288, que establece en su segunda transitoria que los sitios web ecuatorianos que proporcionan un servicio público deben ser accesibles WCAG 2.0 nivel AA hasta el 8 de agosto de 2020 .

\section{Conclusiones}

En la provincia de Loja 46 empresas activas tienen una página web empresarial que trabaja con el dominio.com y domino.com.ec. De estas empresas se pudo determinar que el 57\% utilizan un gestor de contenidos CMS, 17 paginas utilizan WordPress, 15 paginas utilizan Joomla, 1 página utiliza Drupal, 1 Adobe Muse y 12 no disponen de CMS. Con estos datos se puede tener una idea de cómo están diseñadas y estructuradas las páginas web.

La evaluación de las páginas de inicio de los portales web empresariales, arroja un porcentaje bajo en el principio de robustez, y esto deja en evidencia que la página web que incluye tanto la interfaz de usuario y el contenido web, no es totalmente compatible con los navegadores web y los productos de apoyo como; Job Access With Speech JAWS y NonVisual Desktop NVDA, entre otros. En el análisis realizado para determinar los errores en las páginas seleccionadas de los

\footnotetext{
\begin{tabular}{|l|l}
233 & Vol. 5, núm. 3, julio, 2019, pp.212-237 \\
Diego German Salas Paucar, Milton Alfredo Campoverde Molina
\end{tabular}
} 
portales web, la mayor cantidad de estos están presentes en el principio de robustez, coincidiendo con el análisis de porcentaje de cumplimiento las páginas de inicio. Las páginas no están cumpliendo con el reglamento técnico ecuatoriano que establece en la segunda transitoria que para el 8 de agosto de 2020 todo sitio web que preste servicio público debe ser accesible con nivel de conformidad AA de la WCAG 2.0. Los desarrolladores de las empresas deben revisar el código fuente y corregir los errores que están presentes para poder cumplir con las directrices de accesibilidad web.

Las personas con discapacidad en todo el mundo deben poder disfrutar de los mismos derechos y oportunidades que todos los demás, llevar a cabo su vida como ciudadanos de pleno derecho y aportar una valiosa contribución a la sociedad (Unión Internacional de Telecomunicaciones, 2009), en Ecuador la norma NTE INEN-ISO/IEC 40500 fue aprobada el 28 de enero del 2014 y su objetivo es que todos los sitios web de servicio público garanticen la accesibilidad a las personas con algún tipo de discapacidad, para así garantizar la inclusión en estos espacios tecnológicos a todas las personas sin distinción. Es necesario la concienciación sobre este tema a los encargados del desarrollo de las páginas web, para así dar cumplimiento al reglamento técnico ecuatoriano RTE INEN 288.

\section{Referencias Bibliográficas}

AccessMonitor. (2012). Nota técnica del validador AccessMonitor. Retrieved July 3, 2019, from http://www.acessibilidade.gov.pt/accessmonitor/nota_tecnica.html

Acosta, T., \& Luja-Mora, S. (2017). Análisis de la accesibilidad de los sitios web de las universidades ecuatorianas de excelencia. Enfoque UTE,. Quito-Ecuador. Retrieved from http://ingenieria.ute.edu.ec/enfoqueute/index.php/revista/article/view/133/140

Campoverde, M., \& Valverde, L. (2019). Análisis de la accesibilidad de los portales web de las instituciones educativas en la ciudad de Cuenca, Ecuador. Retrieved from http://revistadigital.uce.edu.ec/index.php/CATEDRA/article/view/1646/1618 
Campoverde Molina, M. A., Luján-Mora, S., \& Valverde, L. (2019). Retrospective Analysis of Accessibility of the Educational Web Portals: The Ecuadorian Case (pp. 3774-3783). https://doi.org/10.21125/inted.2019.0961

Consejo Nacional para la Igualdad de Discapacidades. (2019). Estadísticas de Discapacidad Consejo Nacional para la Igualdad de Discapacidades. Retrieved June 14, 2019, from https://www.consejodiscapacidades.gob.ec/estadisticas-de-discapacidad/

Instituto Nacional de Estadística y Censos. (2019). Estadisticas. Retrieved June 14, 2019, from http://www.ecuadorencifras.gob.ec/estadisticas/

Luis Rodríguez V., A. T. de la C. y S. T. C. (2019). Evaluación de la accesibilidad en el sitio web de la Universidad Estatal Península de Santa Elena para personas con discapacidad visual, 1-16. Retrieved from https://www.eumed.net/rev/atlante/2019/02/accesibilidad-sitioweb.html

Lujan-Mora, S. (2019). Accesibilidad Web: Pautas de accesibilidad al contenido web 2.0. Retrieved June 14, 2019, from http://accesibilidadweb.dlsi.ua.es/?menu=pautas-2.0

Luján-Mora, S. (2019a). Accesibilidad Web: WCAG 2.0. Retrieved July 5, 2019, from http://accesibilidadweb.dlsi.ua.es/?menu=wcag-2.0

Luján-Mora, S. (2019b). http://accesibilidadweb.dlsi.ua.es/?menu=hr-revision-automaticas. Retrieved June 17, 2019, from http://accesibilidadweb.dlsi.ua.es/?menu=hr-revisionautomaticas

Mascaraque, E. S. (2009). Herramientas para la evaluación de la accesibilidad Web. Documentación de Las Ciencias de La Información, 32, 245-266. Retrieved from https://dialnet.unirioja.es/servlet/articulo? codigo $=3099165$

Pagnoni, V., \& Mariño, S. (2018). Calidad de contenidos en dominios de educación. Evaluación de la accesibilidad Web mediada por validadores automáticos. Edmetic. https://doi.org/10.21071/edmetic.v8i1.10221 
Registro Oficial. (2016). Registro Oficial No. 686. Retrieved July 4, 2019, from https://www.registroficial.gob.ec/index.php/registro-oficial-web/publicaciones/registrooficial/item/7534-registro-oficial-no-686.html

Servicio Ecuatoriano de Normalización. (2014). NTE INEN-ISO/IEC 40500 tecnología de la información-directrices de accesibilidad para el contenido web del W3C (WCAG) 2.0 (ISO/IEC 40500:2012, IDT) Information Technology-W3C Web Content Accessibility Guidelines (WCAG) 2.0. Quito-Ecuador. Retrieved from https://alemania.embajada.gob.ec/wp-content/uploads/downloads/2018/02/NormativaINEN-de-Accesibilidad.pdf

Servicio Ecuatoriano de Normalización. (2016). Reglamento_INEN_288_Accesibilidad para el Contenido Web.pdf - Google Drive. Retrieved July 5, 2019, from https://drive.google.com/file/d/0Bx14LLo2QyyJdURfOHViRGpCNFU/view

Superintendencia de Compañias Valores y Seguros. (2019). rankingCias. Retrieved June 14, 2019, from https://appscvs.supercias.gob.ec/rankingCias/

TAW. (2019). TAW | Servicios de accesibilidad y movilidad web. Retrieved July 4, 2019, from https://www.tawdis.net/index

Unión Internacional de Telecomunicaciones. (2009). Accesibilidad para todos. Retrieved June 14, 2019, from https://www.itu.int/net/itunews/issues/2009/06/38-es.aspx

WebAIM. (2019). Herramienta de accesibilidad web WAVE. Retrieved July 3, 2019, from http://wave.webaim.org/

WhatCMS. (2019). Detect which CMS a site is using - What CMS? Retrieved July 4, 2019, from https://whatcms.org/

World Wide Web Consortium. (1997). Press Release: W3C Launches International Program Office for WAI. Retrieved June 13, 2019, from https://www.w3.org/Press/IPO-announce 
World Wide Web Consortium. (2008a). Pautas de Accesibilidad para el Contenido Web

(WCAG) 2.0. Retrieved June 14, 2019, from

http://www.sidar.org/traducciones/wcag20/es/

World Wide Web Consortium. (2008b). Web Content Accessibility Guidelines (WCAG) 2.0.

Retrieved June 13, 2019, from https://www.w3.org/TR/WCAG20/

World Wide Web Consortium. (2016). Web Accessibility Evaluation Tools List. Retrieved June

17, 2019, from https://www.w3.org/WAI/ER/tools/ 